\title{
A Neuromotor Device for Reducing Phantom Limb Pain in Individuals with Spinal Cord Injury
}

\author{
Lei Cui ${ }^{1}$, Gonzalo Sepulveda Astudillo ${ }^{1}$ and Garry T. Allison ${ }^{2}$ \\ ${ }^{1}$ Department of Mechanical Engineering, Curtin University, Australia \\ ${ }^{2}$ School of Physiotherapy and Exercise Science, Curtin University, Australia
}

\begin{abstract}
Phantom Limb Pain is a disorder that can be experienced by individuals after amputation or spinal cord injury. In spinal cord injury the paralysis or paresis is often bilateral, thus limiting the application of apparent movement as a therapeutic model for phantom limb pain. This project aimed to develop a robotic rehabilitation device that replicated apparent movement to apply the same therapeutic principles with individuals with lower limb phantom pain that have bilateral paralysis of paresis. The proposed device achieved lower limb planar motion of the knee by a six-bar linkage of a single degree of freedom (DOF). It is driven by a linear actuator while the ankle motion is achieved by a gear motor, reaching an effective $70^{\circ}$ range of motion for both joints. The system features closed loop control using feedback from surface electromyography sensors, limit switches and position sensors with an Arduino microcontroller as the control unit. This device will be used to further our understanding of the disorder and create opportunities for robot aided treatment for individuals with phantom limb pain as a result of spinal cord injury.
\end{abstract}

\section{Introduction}

Spinal cord injury (SCI) is damage to the spinal cord, which could lead to loss of mobility and sensory function. A high proportion of the people with SCI experience neuropathic pain from an area of their body that is paralysed due to central nervous system damage caused by the SCI, rating it as one of the most unmanageable problems [1].

Neuropathic pain occurs in the insensate region below a complete SCI is usually predominated by central nervous system mechanisms in a way similar to phantom limb pain (PLP) following amputations [2]. The person perceives pain from parts of their body that are paralysed in the absence of actual tissue injury, which is not well managed with medications. It has been hypothesized that the illusion of limb movement in individuals with SCI (Moseley et al ) as they think of moving their limb may reduce the symptoms of neuropathic pain, as the mirror technique alleviated the symptoms of PLP in amputees [3].It is likely that any successful therapeutic device relies on the coordination of the imaginary movement (specific motor cortex activation) and the concordant visual feedback of the movement. Such coordination is critical as it has been shown that imaginary movement without visual feedback of the limb moving increases temporary dysthesia or replicates localised pain memory for PLP $[4,5]$.

Since SCI often induces paralysis or paresis of both lower limbs, the neuromotor device needs to be able to move the knee and ankle of the patient in accordance to their thoughts of moving the limb. This effectively creates the illusion that the patient is moving their own leg. This concordant afferent and efferent cortical activation forms the basis of the proven mirror-box rehabilitation methods for individuals with amputations [3].

Lower-limb exoskeletons have been used for various purposes. Ferris et al. [6-9] built a pneumatically powered knee-ankle-foot orthosis, which was used to test this device's mechanical performance during human walking. Chu et al. [10] proposed a hydraulically driven lowerlimb exoskeleton driving the hip, knee, and ankle joints to compensate for the strength and endurance of a human under a payload. Lugris et al. [11] presented an active stance-control knee-ankle-foot orthosis. Cui et al [12] proposed a one active degree-of-freedom closed-loop compliant exoskeleton to enable humans to carry loads during long distance steady locomotion. Dollar and Herr [13] reviewed the state-of-the-art of lower limb exoskeletons in terms of design, actuation, sensory, and control.

However, the aforementioned devices are not able to fulfil this specific task as they either neglect to rotate the ankle joint or do not have the ability to incorporate muscle feedback into the locomotion process. In this paper, we presented a robotic rehabilitation device capable of applying therapeutic principles similar to mirror motion with individuals that have spinal cord 
injury and experience phantom limb pain by simulating limb movement.

This paper is organized as follows. Section 2 introduces the mechanical design of the neuromotor device. Section 3 presents kinematic and stress analyses. Section 4 discusses the system design and implementation of the device. Section 5 concludes the paper.

\section{Overview of mechanical design}

\subsection{Design considerations}

The design considerations defined by the scope of the project are listed in Table 1.

Table 1. Design considerations.

\begin{tabular}{ll}
\hline Customer Requirements & Technical Parameters \\
\hline $\begin{array}{l}\text { Able to accommodate } \\
\text { patients of different } \\
\text { heights }\end{array}$ & Ranging from $1.6 \mathrm{~m}$ to $1.9 \mathrm{~m}$ \\
\hline $\begin{array}{l}\text { Able to withstand the } \\
\text { weight of the leg }\end{array}$ & $\begin{array}{l}\text { Force and stress safety under } \\
\text { the nominal load of } 15 \mathrm{~kg}\end{array}$ \\
\hline $\begin{array}{l}\text { Able to stop at any } \\
\text { instant }\end{array}$ & $\begin{array}{l}\text { Emergency stop } \\
\text { implementation }\end{array}$ \\
\hline Enhanced safety & $\begin{array}{l}\text { Limit switches and software } \\
\text { safety implementation }\end{array}$ \\
\hline Ease of setup and use & $\begin{array}{l}\text { Attached to a rehabilitation } \\
\text { chair }\end{array}$ \\
\hline
\end{tabular}

\subsection{Mechanical design}

We proposed to use a hybrid parallel-serial linkage mechanism to actuate the knee and ankle joints. The knee joint is actuated by a six-bar linkage with a linear actuator drives the knee joint and a servo-motor driven drives the ankle joint, as in Fig. 1.

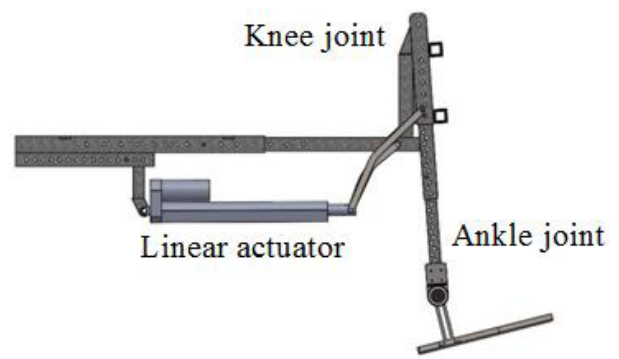

(a) Home position

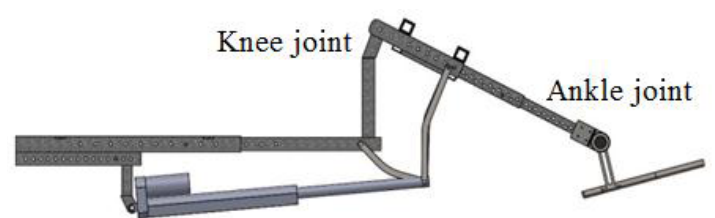

Linear actuator

(b) Extended position

Ankle joint

Figure 1. A hybrid parallel-serial knee-ankle orthosis in home and extended positions

The design features standard sized square hollow section tubing to construct the majority of components supporting the limb. The linear actuator provides an effective range of motion of 70 degrees at the knee joint.

\section{Kinematic and stress analyses}

\subsection{Kinematic Analysis of the knee mechanism}

The six-bar linkage consists of a ternary link and five binary links connected by five revolute joints and one prismatic joint, which is a variation of Stephenson type six-bar linkage [14], as in Fig. 2.

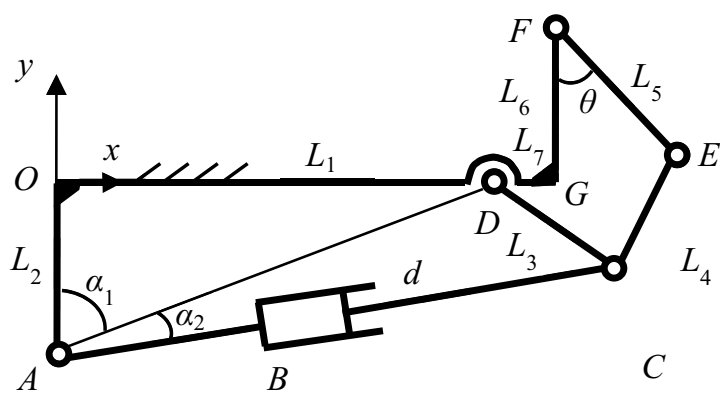

Figure 2. Schematic drawing of the six-bar knee mechanism

The structural parameters are: $O D=L_{1}, O A=L_{2}, C D=L_{3}$, $C E=L_{4}, E F=L_{5}, F G=L_{6}, G D=L_{7}$. The input is $d$, which is the length of $A C$, and the output is knee angle $\theta$, which is the angle between $G F$ and $F E$, as in Fig. 2.

The angle $\alpha_{2}$ between $D A$ and $A C$ can be obtained as

$$
\alpha_{2}=\operatorname{acos}\left(\frac{d^{2}+L_{1}^{2}+L_{2}^{2}-L_{3}^{2}}{2 d \sqrt{L_{1}^{2}+L_{2}^{2}}}\right)
$$

The angle $\alpha_{1}=\operatorname{atan}\left(L_{1} / L_{2}\right)$ is a structural parameter. Hence the coordinates of the joint $C$ are

$$
\begin{aligned}
& C_{x}=d \sin \left(\alpha_{1}+\alpha_{2}\right) \\
& C_{x}=-L_{2}+d \cos \left(\alpha_{1}+\alpha_{2}\right)
\end{aligned}
$$

The link lengths $L_{4}$ and $L_{5}$ constraints transform the problem of finding the knee angle $\theta$ into circle-circle intersection, where the first circle is centred at the joint $\mathrm{C}$ with a radius of $L_{4}$ and the second circle is centred at the joint $\mathrm{F}$ with a radius of $L_{5}$, as in Fig. 3 .

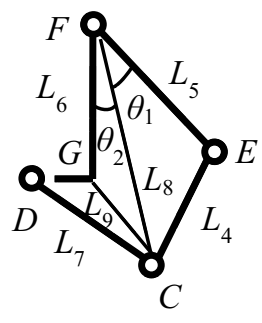

Figure 3. The knee angle $\theta$

It follows that the knee joint $\theta$ is

$\theta=\theta_{1}+\theta_{2}=\operatorname{acos}\left(\frac{L_{8}^{2}+L_{5}^{2}-L_{4}^{2}}{2 L_{5} L_{8}}\right)+\operatorname{acos}\left(\frac{L_{8}^{2}+L_{6}^{2}-L_{9}^{2}}{2 L_{6} L_{8}}\right)$ 
where

$$
\begin{aligned}
& L_{8}=\|C F\|=\sqrt{\left(C_{x}-L_{1}-L_{7}\right)^{2}+\left(C_{y}-L_{6}\right)^{2}} \\
& L_{9}=\|C G\|=\sqrt{\left(C_{x}-L_{1}-L_{7}\right)^{2}+C_{y}^{2}}
\end{aligned}
$$

\subsection{Stress analysis}

We conducted stress analysis in SolidWorks on key parts of the device subject to $15 \mathrm{~kg}$ load, considering the average weight of the calf and foot of a $150 \mathrm{~kg}$ adult is around $150 \mathrm{~kg}$, as in Fig. 4. The weakest component has a safety factor of 1.85 .

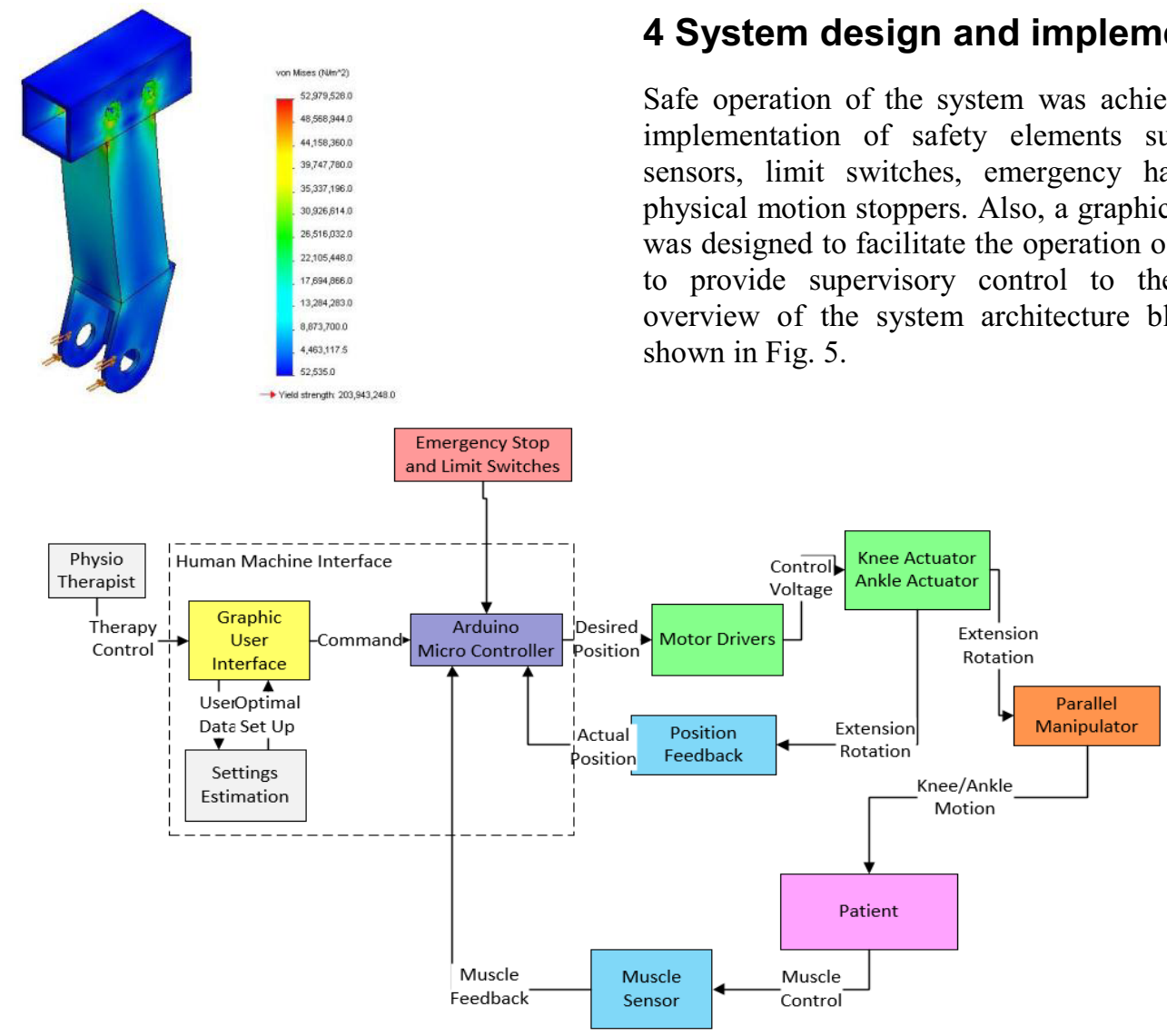

Figure 5. System architecture block diagram

A prototype was built to validate the proposed design, as in Fig. 6.

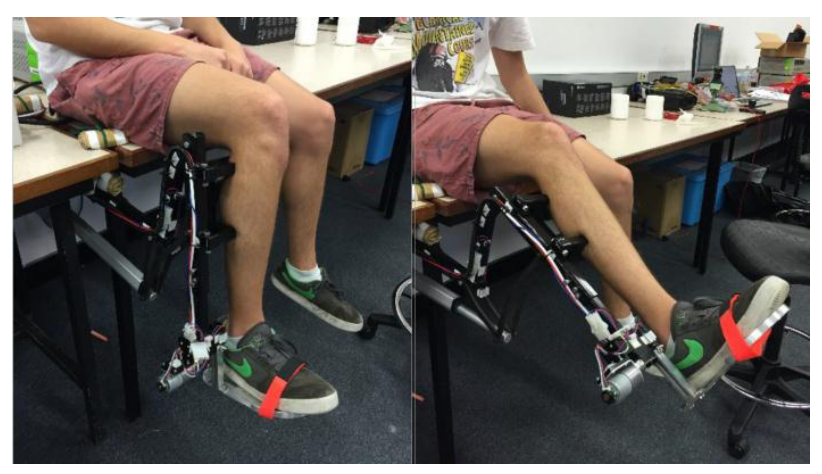

Figure 6. A prototype of the proposed design

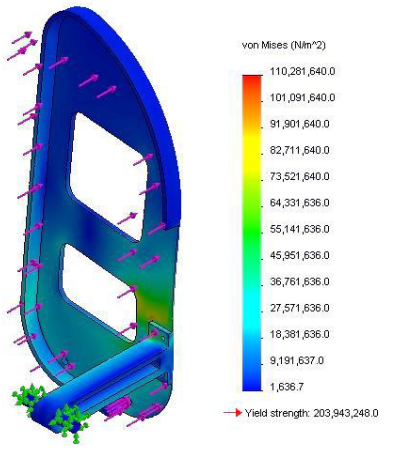

Figure 4. Stress analysis of the base link and the footpad

\section{System design and implementation}

Safe operation of the system was achieved through the

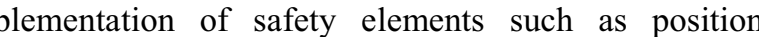
shown in Fig. 5.

We proposed to use diagonal activations where the controller is the EMG signal of the biceps on the upper limb. The individual was instructed to imagine a rope between the hand and ankle and that they act together resulting in the biceps EMG signal initiating the movement, as in Fig. 7.

We tested the device on one healthy subject. Surface EMG electrodes to trigger the muscle feedback function of the system were placed on biceps and the subject bent his forearm to initiate one cycle of rehabilitation training.

The results showed that the motion and speed performance was satisfactory. The lower limb was elevated at a comfortable pace, which was natural and unforced. The joint angles and sEMG sensor signals are shown in Fig. 8. 


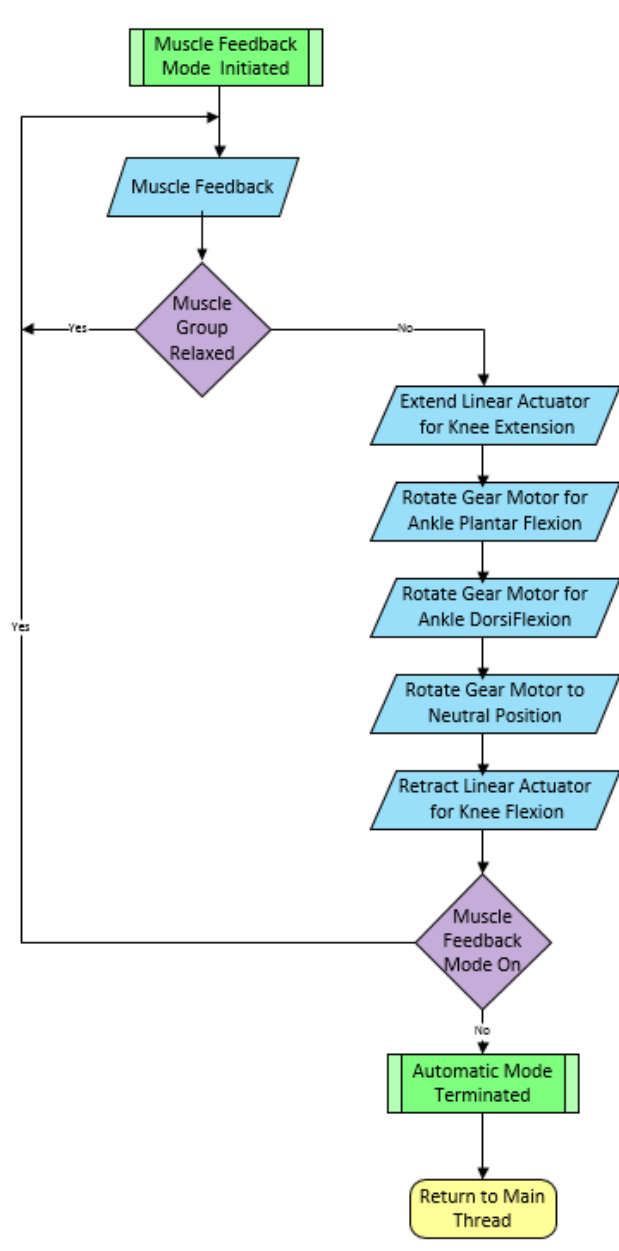

Figure 7. Control flowchart

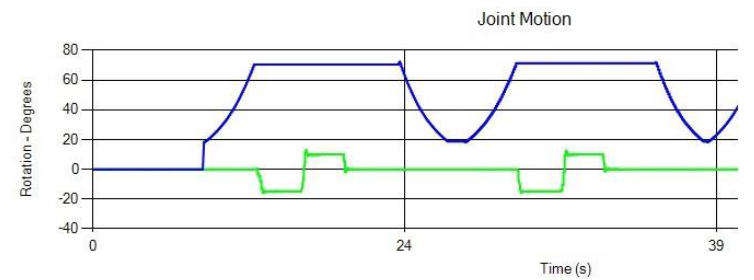

- Ankle Joint — Knee Join

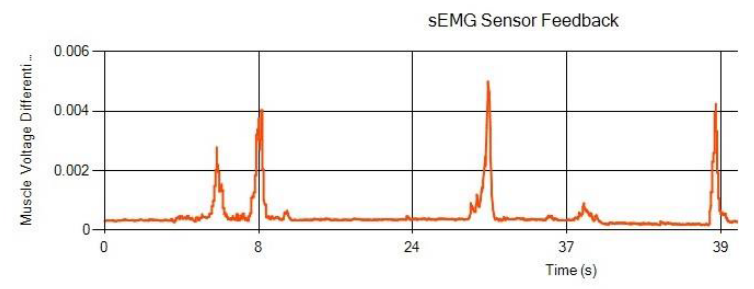

- Muscle Sensor Feedbaci

Figure 8. Knee and ankle joint displacements and the corresponding sEMG signals on the biceps

As can be seen on the joint motion plot, the actuation sequence begins with the ankle actuator, upon reaching the desired position; ankle actuation begins and is followed by knee flexion. Muscle feedback above $2 \mathrm{mV}$ threshold trigger actuation sequence, as seen in both cycles, during this time rehabilitation device only records muscle feedback and signals higher than $2 \mathrm{mV}$ are neglected until cycle is terminated.

\section{Conclusions}

We presented a lower limb rehabilitation system, which introduced planar limb motion to the paralysed limb of patients through a single degree of freedom six-bar linkage for the knee joint and a geared motor for the ankle joint in conjunction with associated control and safety elements. Further development to increase patient comfort in terms of limb support and thorough safety testing would complete the system for patient trials.

\section{References}

1. E. G. Widerström-Noga, E. Felipe-Cuervo, J. G. Broton, R. C. Duncan, and R. P. Yezierski, "Perceived difficulty in dealing with consequences of spinal cord injury," Archives of physical medicine and rehabilitation, vol. 80, pp. 580-586, (1999)

2. H. Flor, T. Elbert, S. Knecht, C. Wienbruch, C. Pantev, N. Birbaumer, et al., "Phantom-limb pain as a perceptual correlate of cortical reorganization following arm amputation," Nature, 375, pp. 482484, (1995)

3. B. L. Chan, R. Witt, A. P. Charrow, A. Magee, R. Howard, P. F. Pasquina, et al., "Mirror therapy for phantom limb pain," New England Journal of Medicine, 357, pp. 2206-2207, (2007)

4. G. L. Moseley, "Using visual illusion to reduce atlevel neuropathic pain in paraplegia," Pain, 130, pp. 294-298, (2007)

5. G. L. Moseley, N. Zalucki, F. Birklein, J. Marinus, J. J. van Hilten, and H. Luomajoki, "Thinking about movement hurts: the effect of motor imagery on pain and swelling in people with chronic arm pain," Arthritis Care \& Research, 59, pp. 623-631, (2008)

6. G. S. Sawicki and D. P. Ferris, "Powered ankle exoskeletons reveal the metabolic cost of plantar flexor mechanical work during walking with longer steps at constant step frequency," Journal of Experimental Biology, 212, (2009)

7. K. E. Gordon and D. P. Ferris, "Learning to walk with a robotic ankle exoskeleton," Journal of Biomechanics, 40, pp. 2636-2644, (2007)

8. P. C. Kao, C. L. Lewis, and D. P. Ferris, "Invariant ankle moment patterns when walking with and without a robotic ankle exoskeleton," Journal of Biomechanics, 43, pp. 203-209, (2010)

9. C. R. Kinnaird and D. P. Ferris, "Medial Gastrocnemius Myoelectric Control of a Robotic Ankle Exoskeleton," IEEE Transactions on Neural Systems and Rehabilitation Engineering, 17, pp. 3137, (2009)

10. A. Chu, H. Kazerooni, and A. Zoss, "On the Biomimetic Design of the Berkeley Lower Extremity Exoskeleton (BLEEX)," in Robotics and Automation, 2005. ICRA 2005. Proceedings of the 2005 IEEE International Conference on, pp. $4345-$ 4352, (2005)

11. J. M. Font-Llagunes, R. Pàmies-Vilà, J. Alonso, and U. Lugrís, "Simulation and design of an active 
orthosis for an incomplete spinal cord injured subject," Procedia IUTAM, vol. 2, pp. 68-81, (2011)

12. Z. Shen, M. White, G. T. Allsion, and L. Cui, "Single Degree-of-Freedom Leg Exoskeleton Assisting Human Locomotion," presented at the IEEE/RSJ International Conference on Intelligent Robots and Systems, Deajeon, South Korea, (2016)
13. A. M. Dollar and H. Herr, "Lower Extremity Exoskeletons and Active Orthoses: Challenges and State-of-the-Art," IEEE Transactions on Robotics, 24, pp. 144-158, (2008)

14. K.-L. Ting and X. Dou, "Classification and branch identification of Stephenson six-bar chains," Mechanism and Machine Theory, 31, pp. 283-295, (1996) 